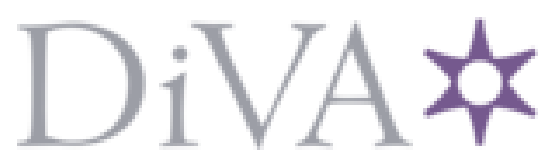

http://www.diva-portal.org

\title{
Postprint
}

This is the accepted version of a paper presented at 2013 IEEE 24th Annual International Symposium on Personal, Indoor, and Mobile Radio Communications, PIMRC 2013; London; United Kingdom; 8 September 2013 through 11 September 2013.

Citation for the original published paper:

Shalmashi, S., Miao, G., Slimane, B. (2013)

Interference management for multiple device-to-device communications underlaying cellular networks.

In: 2013 IEEE 24th International Symposium on Personal Indoor and Mobile Radio

Communications (PIMRC) (pp. 223-227). IEEE

http://dx.doi.org/10.1 109/PIMRC.2013.6666135

N.B. When citing this work, cite the original published paper.

Permanent link to this version:

http://urn.kb.se/resolve?urn=urn:nbn:se:kth:diva-128565 


\section{Interference Management for Multiple Device-to- Device Communications Underlaying Cellular Networks}

IEEE International Symposium on Personal, Indoor and Mobile Radio Communications (PIMRC), London, UK, September 2013

(C) 2013 IEEE. Personal use of this material is permitted. Permission from IEEE must be obtained for all other uses, in any current or future media, including reprinting/republishing this material for advertising or promotional purposes, creating new collective works, for resale or redistribution to servers or lists, or reuse of any copyrighted component of this work in other works.

\section{SERVEH SHALMASHI, GUOWANG MIAO, SLIMANE BEN SLIMANE}

This is an author produced version of the paper.

Access to the published version may require subscription.

Published with permission from: IEEE 


\title{
Interference Management for Multiple Device-to-Device Communications Underlaying Cellular Networks
}

\author{
Serveh Shalmashi, Guowang Miao, and Slimane Ben Slimane \\ Department of Communication Systems (CoS) \\ School of Information and Communication Technology (ICT) \\ KTH Royal Institute of Technology, Stockholm, Sweden \\ \{serveh, guowang, slimane\}@kth.se
}

\begin{abstract}
We study the problem of interference management for device-to-device (D2D) communications where multiple D2D users may coexist with one cellular user. The problem is to optimize the transmit power levels of D2D users to maximize the cell throughput while preserving the signal-to-noise-plusinterference ratio (SINR) performance for the cellular user. This is the so-called multi rate power control problem. We investigate the problem under two assumptions, the availability of the instantaneous or average channel state information (CSI) at the base station. In the first case, D2D transmit power levels adapt to fast fading, whereas in the second case, they only adapt to slow fading. In the latter assumption, the cellular user has a maximum outage probability requirement. With numerical results, we study the trade-off between the signaling overhead, that is frequent CSI feedbacks, and the overall system performance, that is the maximum achievable cell capacity, for D2D communications underlying cellular networks.
\end{abstract}

\section{INTRODUCTION AND RELATED WORK}

In conventional cellular networks, mobile users communicate to each other via a base station. However, when mobile transmitters and receivers are close, it can be beneficial if they communicate directly, i.e., using the so-called device-to-device (D2D) communication mode [1]. Exploiting this proximity gain leads to a higher power efficiency and data rate for both the network and mobile users [1]-[3]. Users in the D2D mode can transmit either in a separate frequency band or via spectrum sharing as long as the cellular users are protected against interference. In this work, our focus is on spectrum sharing using the uplink resources to increase cell capacity.

The achievable capacity gain of the network in the presence of $\mathrm{D} 2 \mathrm{D}$ communications is assured by power control and proper mode selection. These schemes manage the interference from the D2D users to the cellular users and vice versa. The work in [4] and [5] control the interference of the D2D links to the cellular users by limiting the maximum transmit power of the D2D users. Authors in [6] aim at minimizing the maximum interferences from both cellular users to D2D users and from

Part of this work has been performed in the framework of the FP7 project ICT-317669 METIS, which is partly funded by the European Union. The authors would like to acknowledge the contributions of their colleagues in METIS, although the views expressed are those of the authors and do not necessarily represent the project.
D2D users to cellular users by means of power control. In [7], authors employ the interference-limited area knowledge for $\mathrm{D} 2 \mathrm{D}$ receivers to maximize the network capacity with multiuser MIMO, and any cellular users in the vicinity of the interference-limited area is not scheduled. Mode selection that takes into account the effect of interference in spectrum sharing has been addressed in [8], where the goal is to find the best mode to maximize sum rate. Authors in [9] combine the mode selection with power control.

The existing scenarios in literature have investigated the coexistence of only one D2D user with a cellular user in a single frequency band [4], [5], [9], [10], while by proper power control and efficient mode selection multiple D2D users can communicate in one cell for higher network throughput. Therefore, in our work, we assume that more than one D2D user may reuse the spectrum to improve capacity. We fix the cellular user's transmit power and optimize the transmit powers of active D2D users. The cellular user has a quality-ofservice (QoS) requirement. Furthermore, we assume that the received interference power from the D2D users at the base station should be the same for all users. This assumption forces the D2D users close to the cell center to communicate via the base station in order to decrease the received interference. We formulate this problem as a multi-rate power control problem under the assumption of the availability of instantaneous or statistical channel state information (CSI). In most power control schemes, the powers are updated every instance the fading state of the channel changes [11]. This is the underlying assumption in our first scenario, where we obtain the exact maximum number of active D2D users for the maximum cell throughput while preserving the cellular user's QoS requirement. However, this scenario requires frequent CSI feedbacks in order to update the powers, which leads to significant signaling overhead and power consumption. Therefore, in the second scenario, the amount of required information for D2D power allocations is decreased. We assume only statistical CSIs based on the location of D2D users. Hence, the transmit power updates only follow the path loss while certain outage probability is allowed for the cellular user. Based on these two formulations, we investigate the trade-off between the 
available CSI information, the number of served D2D links, and the maximum achievable cell capacity.

\section{SySTEM MOdEL}

We consider a single-cell cellular network with one cellular user (CUE) communicating with the base station. In addition, there are $K$ other users in the cell that are in the D2D mode and can communicate directly with their receivers. The D2D users utilize the uplink resources of the cellular network. We assume more than one D2D user may transmit simultaneously. The overall network performance depends on the transmit powers of active users. In this model, the transmit power of the cellular user is fixed, but the power levels of D2D users are optimized to control the interference. To this end, the received interference power from all D2D users are assumed to be the same at the base station. This means that the D2D users that are far away from the base station can communicate directly without causing much interference to the infrastructure link and the ones that are close to the cell center may communicate via the base station instead.

To model the active D2D users, we define a binary random variable $x_{k} \in\{0,1\}, k \in\{1, \ldots, K\}$, where $x_{k}=1$ corresponds to the event that the $k$ th D2D user is active, otherwise $x_{k}=0$. Let $G_{i j}$ denote the instantaneous channel gain from the $i$ th transmitter to the $j$ th receiver with $i, j \in\{0,1, \ldots, K\}$, where $i, j=0$ corresponds to the cellular user and the base station, and the D2D users otherwise. The instantaneous channel gain accounts for the path loss, $\bar{g}_{i j}$, and Rayleigh fading, $\left|h_{i j}\right|^{2}$, with $\mathbb{E}\left[\left|h_{i j}\right|^{2}\right]=2 \sigma^{2}=1$, i.e.,

$$
G_{i j}=\bar{g}_{i j}\left|h_{i j}\right|^{2} \text {. }
$$

Define the cellular user's and the $k$ th D2D user's signal-tonoise-plus-interference ratio (SINR) as

$$
\begin{aligned}
\gamma_{0} & =\frac{p_{0} G_{00}}{\sum_{k=1}^{K} x_{k} p_{k} G_{k 0}+N}, \\
\gamma_{k} & =\frac{x_{k} p_{k} G_{k k}}{\sum_{k^{\prime}=1, k^{\prime} \neq k}^{K} x_{k^{\prime}} p_{k^{\prime}} G_{k^{\prime} k}+p_{0} G_{0 k}+N},
\end{aligned}
$$

where $p_{j}, j \in\{0,1, \ldots, K\}$, are the transmit powers, and $N$ is the receiver noise power. Then, the corresponding achievable rates are $R_{0}=\log _{2}\left(1+\gamma_{0}\right)$ for the cellular link and $R_{k}=$ $\log _{2}\left(1+\gamma_{k}\right)$ for D2D link $k$ measured in $[\mathrm{bps} / \mathrm{Hz}]$, and the objective is

$$
\max _{p_{k}, x_{k}} R_{0}+\sum_{k=1}^{K} x_{k} R_{k} .
$$

\section{PROBlEM Formulation}

We formulate the problem based on two different constraints, namely, instantaneous SINR constraint, and outage constraint. The former requires that the instantaneous channel gains of all D2D users to be available at the base station in every transmission time slot, thus, imposing a high signaling overhead among network entities. The latter, however, only requires average channel gains of the D2D users that only depend on the path loss. Since the positions of D2D users do not change rapidly, frequent channel feedbacks are not necessary and signaling overhead is reduced.

\section{A. Instantaneous SINR constraint}

In order to protect the cellular user, a pre-determined threshold, $\gamma_{0}^{\text {th }}$, for the instantaneous SINR should be met, i.e.,

$$
\gamma_{0} \geq \gamma_{0}^{\text {th }} .
$$

As there is a limit on the transmission power of mobile devices, we also have

$$
0 \leq p_{k} \leq P_{\max }, \quad \forall k \in\{1, \ldots, K\}
$$

We assume that the CUE transmits with its maximum power, i.e., $p_{0}=P_{\max }$. In order to control the interference, we assume that the instantaneous received power, $\bar{p}$, at the base station from all the active D2D users are the same and we have

$$
\bar{p}=p_{k} G_{k 0}, \quad \forall k \in\{1, \ldots, K\} .
$$

Here, the common received power accounts for the effects of path loss and fading. Let $\mathcal{A} \triangleq\left\{k \mid x_{k}=1, k \in\{1 \ldots, K\}\right\}$ define the set of active D2D links, with cardinality $|\mathcal{A}|=$ $L$. First assume $L$ is known. The SINR constraint in (4) can equivalently be written as

$$
\frac{p_{0} G_{00}}{\bar{p} \sum_{k=1}^{K} x_{k}+N}=\frac{p_{0} G_{00}}{\bar{p} L+N} \geq \gamma_{0}^{\text {th }} .
$$

Therefore, the maximum tolerable received interference power from each D2D transmitter is upper bounded by

$$
\bar{p} \leq \frac{1}{L}\left(\frac{p_{0} G_{00}}{\gamma_{0}^{\text {th }}}-N\right) .
$$

Substituting (8) in (6) for $k \in \mathcal{A}$, we obtain the transmit powers of the D2D users.

The remaining question is the optimal $L$. The maximum acceptable $\bar{p}$ from (8) is a function of $L$, the number of active D2D users. Therefore, in the next step, the number of active D2D links that maximizes (3) is found. The optimal solution to the multi-rate power control problem is obtained by a brute-force enumeration algorithm. The enumeration algorithm works as follows:

1) First, all possible combinations of active and inactive D2D links are determined. At most $K$ D2D users can be admitted which use the CUE's spectrum. The total number of combinations is $2^{K}$.

2) In the next step, we sort all combinations in a descending order, based on the number of active links.

3) Then, for each combination of active users, the power control problem in (5) and (8) is solved. Using the obtained transmit powers, rate of the CUE and every D2D links are determined from their resulting SINRs in (1) and (2).

4) Finally, among all possible combinations, the one which maximizes the cell sum rate in (3) is chosen as the optimal solution.

There are instances when, without any interference from the 


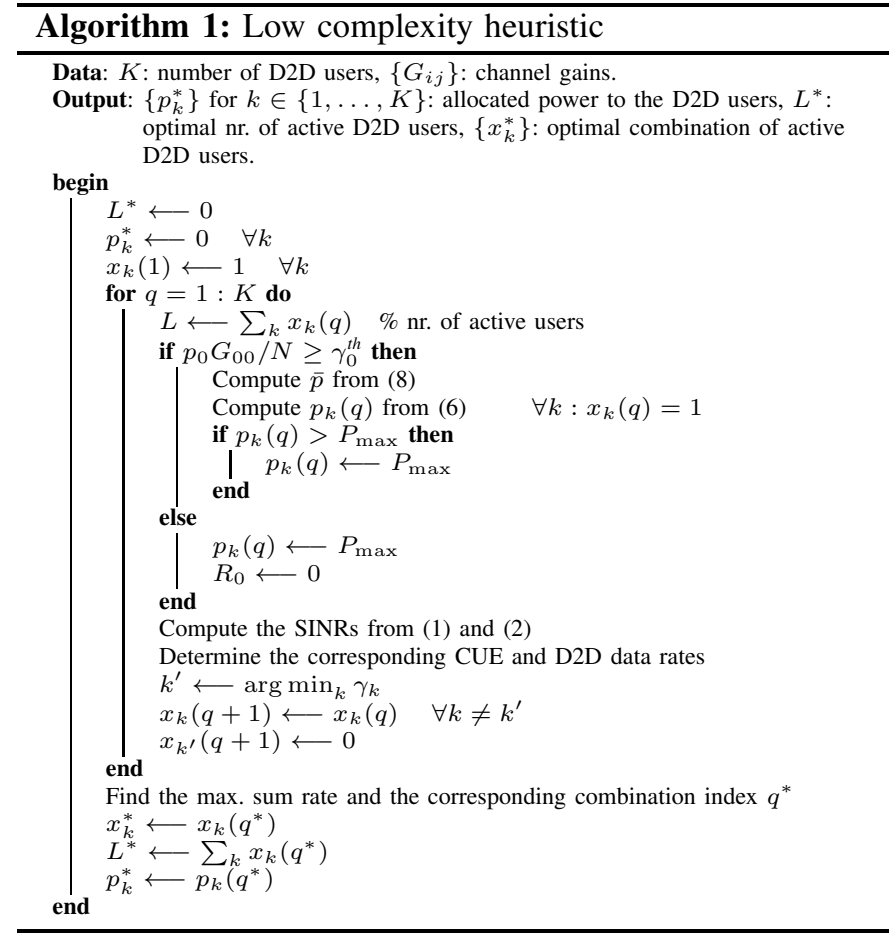

D2D users, the cellular user is in outage, i.e.,

$$
\frac{p_{0} G_{00}}{N} \leq \gamma_{0}^{\text {th }}
$$

In such a case, the achievable rate of the CUE is zero. As we do not consider any QoS requirements for D2D users, they can be active with maximum power $p_{k}=P_{\max }$.

This approach enumerates over all $2^{K}$ possible cases of active D2D links which is difficult to handle if the number of D2D users is high. Therefore, we develop a heuristic algorithm, depicted in Algorithm 1, in order to reduce the complexity and solve the problem in a manageable time. In this algorithm, the power control multi-rate problem is initially solved for $L=K$ active links. In the next step, the D2D user with the lowest SINR is dropped [12] and the same problem is solved with the remaining $L=K-1$ active links. In this way, the algorithm only requires $K$ iterations in total, resulting in a very low complexity. However, the low complexity comes at the price of obtaining a sub-optimal solution. In section IV, the performance of the sub-optimal algorithm is compared to the optimal enumeration approach.

\section{B. Outage constraint}

In this section, we assume that the power updates of the D2D transmitters are not instantaneous and do not follow fast fading. That is, the power allocations can only compensate for slow fading, and we have

$$
\bar{p}=p_{k} \bar{g}_{k 0} \quad \forall k \in\{1, \ldots, K\} .
$$

Hence, the received SINR of the cellular user is

$$
\frac{p_{0} G_{00}}{\bar{p} \sum_{k=1}^{K} x_{k}\left|h_{k 0}\right|^{2}+N}=\frac{p_{0} G_{00}}{\bar{p} \sum_{\ell=1}^{L}\left|h_{\ell 0}\right|^{2}+N} \geq \gamma_{0}^{\text {th }},
$$

where $L$ is the number of active D2D users. Then, the outage probability for the cellular user is

$$
P_{\text {out }}=\operatorname{Pr}\left(\frac{p_{0} G_{00}}{\bar{p} \sum_{\ell=1}^{L}\left|h_{\ell 0}\right|^{2}+N} \leq \gamma_{0}^{\text {th }}\right) .
$$

To compute the outage probability, we first introduce the following lemma [11].

Lemma 1. Suppose $z_{c}$ and $z_{1}, \ldots, z_{L}$ are independent exponentially distributed random variables with means $1 / \lambda_{c}$ and $1 / \lambda_{\ell}$ for $\ell=1, \ldots$. Then, we have

$$
\operatorname{Pr}\left(\frac{z_{c}}{\sum_{\ell=1}^{L} z_{\ell}+1} \leq b\right)=1-e^{-b \lambda_{c}} \prod_{\ell=1}^{L} \frac{1}{1+b \frac{\lambda_{c}}{\lambda_{\ell}}} .
$$

Proof: Setting $Q=b\left(\sum_{\ell=1}^{L} z_{\ell}+1\right)$, we have

$$
\begin{aligned}
\operatorname{Pr}\left(z_{c} \geq Q\right) & =\mathbb{E}\left[\operatorname{Pr}\left(z_{c} \geq Q\right) \mid z_{1} \ldots z_{L}\right] \\
& =\mathbb{E}\left[e^{-\lambda_{c} Q} \mid z_{1} \ldots z_{L}\right] \\
& =\int_{z_{1}=0}^{\infty} \cdots \int_{z_{L}=0}^{\infty} e^{-\lambda_{c} Q} \prod_{\ell=1}^{L} \lambda_{\ell} e^{-\lambda_{\ell} z_{\ell}} d z_{1} \cdots d z_{L} \\
& =e^{-b \lambda_{c}} \prod_{\ell=1}^{L} \int_{z_{\ell}=0}^{\infty} \lambda_{\ell} e^{-\left(b \lambda_{c}+\lambda_{\ell}\right) z_{\ell}} d z_{\ell} \\
& =e^{-b \lambda_{c}} \prod_{\ell=1}^{L} \frac{\lambda_{\ell}}{b \lambda_{c}+\lambda_{\ell}} .
\end{aligned}
$$

Therefore,

$$
\begin{aligned}
\operatorname{Pr}\left(z_{c} \leq Q\right) & =1-\operatorname{Pr}\left(z_{c} \geq Q\right) \\
& =1-e^{-b \lambda_{c}} \prod_{\ell=1}^{L} \frac{1}{1+b \frac{\lambda_{c}}{\lambda_{\ell}}} .
\end{aligned}
$$

The outage probability in (12) can be rewritten as

$$
P_{\text {out }}=\operatorname{Pr}\left(\frac{\Gamma_{0}}{\sum_{\ell=1}^{L} \Gamma_{\ell 0}+1} \leq \gamma_{0}^{\text {th }}\right)
$$

where

$$
\Gamma_{0}=\frac{p_{0} G_{00}}{N}, \quad \Gamma_{\ell}=\frac{\bar{p}\left|h_{\ell 0}\right|^{2}}{N} .
$$

Both $\Gamma_{0}$ and $\Gamma_{\ell}$ are exponentially distributed with density functions

$$
f_{0}(\gamma)=\frac{1}{\bar{\gamma}_{0}} e^{-\frac{\gamma}{\bar{\gamma}_{0}}}, \quad f_{\ell}(\gamma)=\frac{1}{\bar{\gamma}_{d}} e^{-\frac{\gamma}{\bar{\gamma}_{d}}},
$$

and means

$$
\begin{aligned}
& \bar{\gamma}_{0}=\mathbb{E}\left[\Gamma_{0}\right]=\frac{2 \sigma^{2} p_{0} \bar{g}_{00}}{N}, \\
& \bar{\gamma}_{d}=\mathbb{E}\left[\Gamma_{\ell}\right]=\frac{2 \sigma^{2} \bar{p}}{N} .
\end{aligned}
$$


TABLE I

SIMULATION PARAMETERS.

\begin{tabular}{lcc}
\hline Description & Parameter & Value \\
\hline Max. UE TX power & $P_{\max }$ & $24 \mathrm{dBm}$ \\
Cell Radius & $R$ & $200 \mathrm{~m}$ \\
CUE SINR threshold & $\gamma_{0}^{\text {th }}$ & $0,2,4,6 \mathrm{~dB}$ \\
Noise power & $N$ & $-107 \mathrm{dBm}$ \\
Carrier frequency & $f_{c}$ & $2 \mathrm{GHz}$ \\
System Bandwidth & $B$ & $5 \mathrm{MHz}$ \\
D2D pair distance & $D^{d}$ & $4-40 \mathrm{~m}$ \\
Min. D2D Tx distance from BS & $D_{\min }^{\prime}$ & $10 \mathrm{~m}$ \\
Min. D2D CUE distance from BS & $D_{\min }$ & $10 \mathrm{~m}$ \\
Monte-Carlo simulation runs & $\mathrm{MC}$ & 5000 \\
\hline
\end{tabular}

Then, based on Lemma 1 , the outage probability is derived as

$$
P_{\text {out }}=1-e^{-\frac{\gamma_{0}^{\text {th }}}{\bar{\gamma}_{0}}}\left(\frac{\frac{\bar{\gamma}_{0}}{\gamma_{0}^{\text {th }}}}{\bar{\gamma}_{d}+\frac{\bar{\gamma}_{0}}{\gamma_{0}^{\text {th }}}}\right)^{L} .
$$

To allow D2D users be active in the cell based only on the average channel gains, a certain outage probability for the base station should be ensured, i.e.,

$$
P_{\text {out }} \leq p_{\text {out }}^{\text {th }}
$$

with $p_{\text {out }}^{\text {th }}>0$. Using (16)-(19), we can calculate an upper bound on the allowable received interference power, $\bar{p}$, at the base station from each active D2D user as

$$
\bar{p} \leq \frac{p_{0} \bar{g}_{00}}{\gamma_{0}^{\text {th }}}\left[\left(\frac{e^{-\frac{\gamma_{0}^{\text {th }}}{\bar{\gamma}_{0}}}}{1-p_{\text {out }}^{\text {th }}}\right)^{\frac{1}{L}}-1\right]^{+},
$$

where $[z]^{+}=\max \{z, 0\}$. Now based on (5) and (10), the transmit power for all active D2D users is determined.

To find the optimal solution for the problem (3) based on the availability of average channel gains with the constraints (5) and (19), we can follow a similar enumeration approach as before with the corresponding power update steps in (20) and (10).

\section{Performance Evaluation}

We evaluate the performance of our model as described in Sec. II by means of Monte-Carlo simulations. We consider a circular cell layout with radius $R$. The base station is located in the center of the cell. In each realization, one CUE and $K$ D2D transmitter-receiver pairs are generated randomly with uniform distribution over the cell area. The channel model accounts for the effects of path loss and multi-path fading. The path loss model is implemented based on the model described in [13]. Simulation parameters are given in Table I.

In Fig. 1, we compare the performance of the heuristic algorithm in Algorithm 1 to the brute-force enumeration approach. It can be seen that the heuristic algorithm follows the brute-force enumeration closely with a significantly lower complexity. The figure also depicts the increase in the cell sum rate compared to the case where there is no D2D

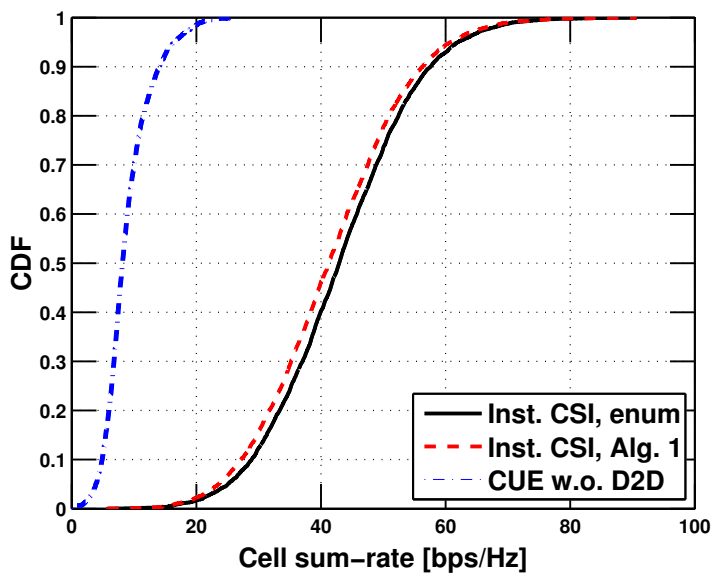

Fig. 1. Performance of the heuristic approach compared to the enumeration with $K=10$ and $\gamma_{0}^{\text {th }}=2 \mathrm{~dB}$.

communications. Fig. 2 shows the average number of active D2D users versus different number of available D2D users $(K)$ in the cell for different approaches. It is observed that with a higher number of available users, more D2D links can be active without degrading the CUE's performance. The gap between the performance based on the instantaneous CSI and the average CSI stems from the fact that in the latter case, due to the lack of instantaneous knowledge, the D2D users' transmit powers are overestimated resulting in fewer number of active users. Similar results hold for the average cell sum rate shown in Fig. 3 for two extreme cases of cellular QoS constraints. Fig. 4 shows the cell sum rate under different CUE's SINR thresholds for the setup with instantaneous and average CSI. Here, we can observe a trade-off between the performance of the network and the amount of available CSI. Better performance requires more frequent CSI feedbacks and consequently high signaling overhead. In Fig. 5, we can see the average cell sum rate under different probabilities of outage thresholds for the cellular user when only average CSI is available. The more restriction on the outage probability, the less increase in the cell capacity compared to the scenario without D2D communications.

\section{CONCLUSiOns}

We have studied the coexistence of multiple D2D users with one cellular user. We have formulated the problem of sum rate maximization under SINR constraint of the cellular user. In order to protect the cellular receiver, we have assumed that the received interference power from all D2D users at the base station are the same. This assumption encourages the D2D users that create less interference to transmit when they reuse the uplink resources of the network. The D2D users closer to the cell center communicate via the base station as they cause more interference. We have investigated the interference management schemes with the availability of either instantaneous CSI or average CSI. The simulation results have shown that multiple D2D links can share the spectrum with one cellular link without degrading its performance. We have also observed the trade-off between the signaling overhead 


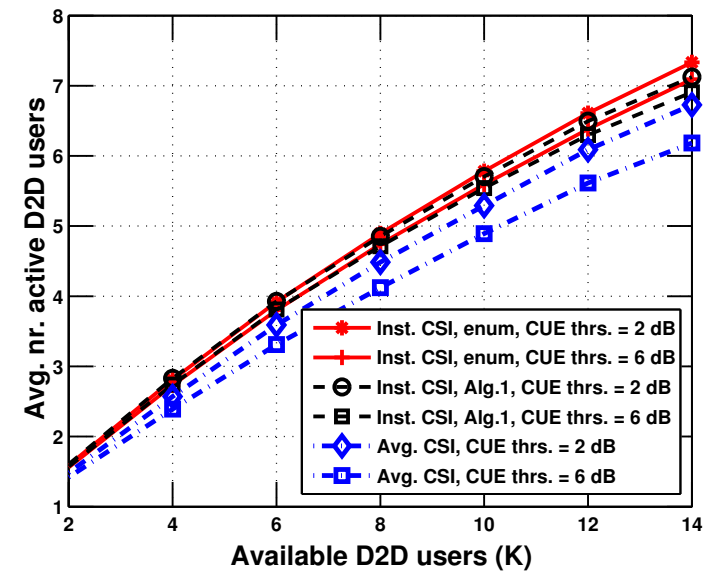

Fig. 2. Average nr. of active D2D users vs. nr. of D2Ds in the cell for $\gamma_{0}^{\text {th }}=2 \mathrm{~dB}$ and $p_{\text {out }}^{\text {th }}=10 \%$.

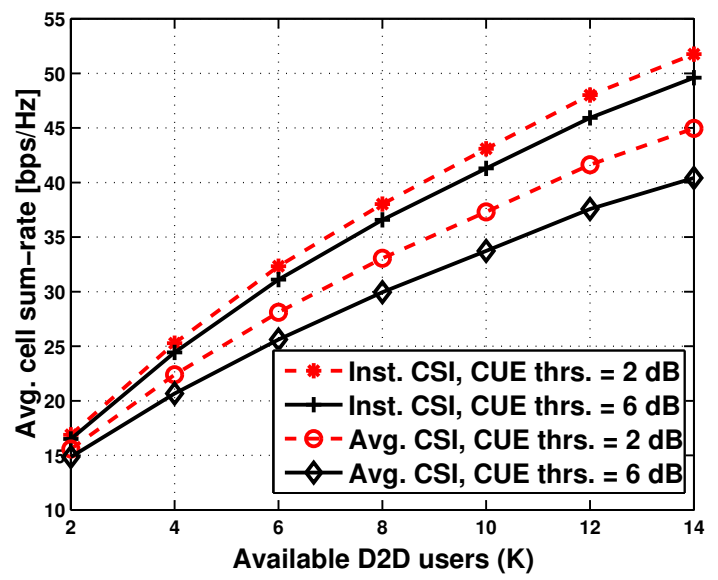

Fig. 3. Average cell sum rate based on average and instantaneous CSI for $\gamma_{0}^{\text {th }}=0,6 \mathrm{~dB}$ and $p_{\text {out }}^{\text {th }}=10 \%$.

and the performance of the network. In our future work, we will try to improve the performance of the interference control scheme when only the average CSI is available in a multi-cell scenario.

\section{REFERENCES}

[1] L. Lei, Z. Zhong, C. Lin, and X. Shen, "Operator controlled deviceto-device communications in lte-advanced networks," IEEE Wireless Commun. Mag., vol. 19, no. 3, pp. 96-104, Jun. 2012.

[2] K. Doppler, M. Rinne, C. Wijting, C. Ribeiro, and K. Hugl, "Device-todevice communication as an underlay to lte-advanced networks," IEEE Commun. Mag., vol. 47, no. 12, pp. 42-49, Dec. 2009.

[3] G. Fodor, E. Dahlman, G. Mildh, S. Parkvall, N. Reider, G. Miklós, and Z. Turányi, "Design aspects of network assisted device-to-device communications," IEEE Commun. Mag., vol. 50, no. 3, pp. 170-177, Mar. 2012.

[4] P. Jänis, C. Yu, K. Doppler, C. Ribeiro, C. Wijting, K. Hugl, O. Tirkkonen, and V. Koivunen, "Device-to-device communication underlaying cellular communications systems," IEEE Trans. Inf. Theory, vol. 2, no. 3, pp. 169-178, Mar. 2009.

[5] C. H. Yu, O. Tirkkonen, K. Doppler, and C. B. Ribeiro, "On the performance of device-to-device underlay communication with simple power control," in Proc. IEEE Vehicular Technology Conf. (VTC), Apr. 2009, pp. $1-5$.

[6] P. Jänis, V. Koivunen, C. Ribeiro, J. Korhonen, K. Doppler, and K. Hugl, "Interference-aware resource allocation for device-to-device radio underlaying cellular networks," in Proc. IEEE Vehicular Technology Conf. (VTC), Apr. 2009, pp. 1-5.

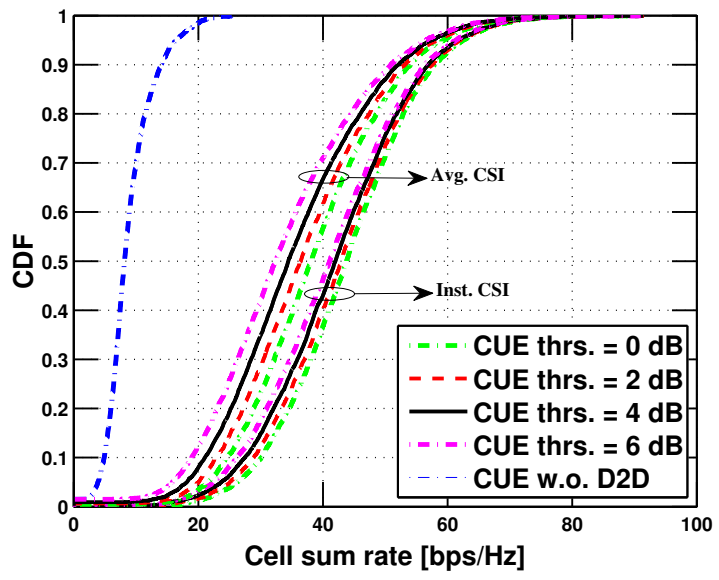

Fig. 4. CDF of cell sum rate based on average and instantaneous CSI for different $\gamma_{0}^{\text {th }}$ and $p_{\text {out }}^{\text {th }}=10 \%$

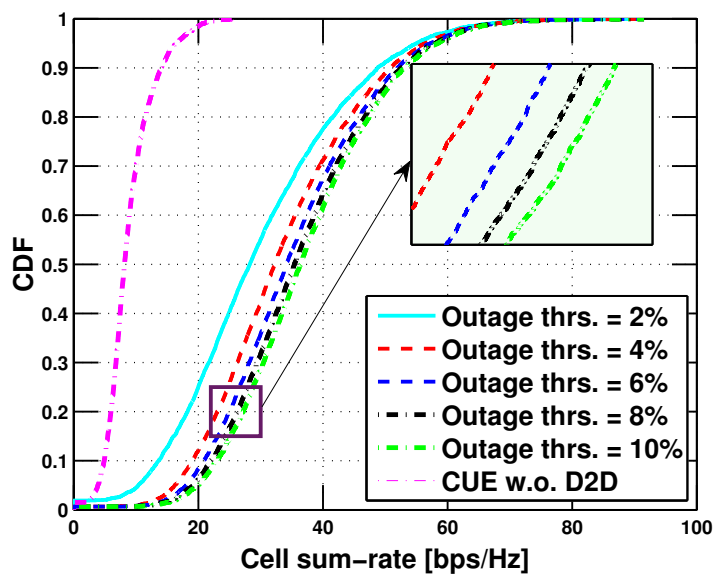

Fig. 5. CDF of cell sum rate based on average CSI for $K=10$, different values of $p_{\text {out }}^{\text {th }}$, and $\gamma_{0}^{\text {th }}=2 \mathrm{~dB}$.

[7] H. Min, J. Lee, S. Park, and D. Hong, "Capacity enhancement using an interference limited area for device-to-device uplink underlaying cellular networks," IEEE Trans. Wireless Commun., vol. 10, no. 12, pp. 39954000, Dec. 2011.

[8] K. Doppler, C. Yu, C. Ribeiro, and P. Jänis, "Mode selection for deviceto-device communication underlaying an lte-advanced network," in Proc. IEEE Wireless Commun. Network. Conf. (WCNC), Apr. 2010, pp. 1-6.

[9] C. Yu, K. Doppler, C. Ribeiro, and O. Tirkkonen, "Resource sharing optimization for device-to-device communication underlaying cellular networks," IEEE Trans. Wireless Commun., vol. 10, no. 8, pp. 27522763, Aug. 2011.

[10] M. Zulhasnine, S. Changcheng, and A. Srinivasan, "Efficient resource allocation for device-to-device communication underlaying lte network," in Proc. IEEE Int. Conf. on Wireless and Mobile Computing, Networking, and Commun. (WiMob), Oct. 2010, pp. 368-375.

[11] S. Kandukuri and S. Boyd, "Optimal power control in interferencelimited fading wireless channels with outage-probability specifications," IEEE Trans. Wireless Commun., vol. 1, no. 1, pp. 46-55, Jan. 2002.

[12] M. Andersin, Z. Rosberg, and J. Zander, "Gradual removals in cellular pcs with constrained power control and noise," in Proc. IEEE Int. Symp. on Personal, Indoor, Mobile Radio Commun. (PIMRC), Sep. 1995, pp. $56-60$.

[13] H. Xing and S. Hakola, "The investigation of power control schemes for a device-to-device communication integrated into ofdma cellular system," in Proc. IEEE Int. Symp. on Personal, Indoor, Mobile Radio Commun. (PIMRC), Sep. 2010, pp. 1775-1780. 